\title{
ON THE BOUNDEDNESS OF SOLUTIONS TO ELLIPTIC VARIATIONAL INEQUALITIES
}

\author{
PATRICK WINKERT
}

\begin{abstract}
In this paper we present global a priori bounds for a class of variational inequalities involving general elliptic operators of second-order and terms of generalized directional derivatives. Based on Moser's and De Giorgi's iteration technique we prove the boundedness of solutions of such inequalities under certain criteria on the set of constraints. In our proofs we also use the localization method with a certain partition of unity and a version of a multiplicative inequality estimating the boundary integrals. Some sets of constraints satisfying the required conditions are stated as well.
\end{abstract}

\section{INTRODUCTION}

Given a bounded domain $\Omega \subset \mathbb{R}^{N}, N>1$, with Lipschitz boundary $\partial \Omega$, we consider the following problem: Find $u \in K$ such that

$$
\begin{aligned}
& \int_{\Omega} A(x, u, \nabla u) \cdot \nabla(v-u) d x+\int_{\Omega} F(x, u, \nabla u)(v-u) d x \\
& +\int_{\Omega} j_{1}^{\circ}(x, u ; v-u) d x+\int_{\partial \Omega} j_{2}^{\circ}(x, u ; v-u) d \sigma \geq 0,
\end{aligned}
$$

for all $v \in K$, where $K$ is a subset of a Banach space $V$ (will be specified below) and $j_{k}^{\circ}(x, s ; r)(k=1,2)$ denotes the generalized directional derivative of a locally Lipschitz function $s \mapsto j_{k}(x, s)$ at $s$ in the direction $r$. The maps $A: \Omega \times \mathbb{R} \times \mathbb{R}^{N} \rightarrow$ $\mathbb{R}^{N}$ and $F: \Omega \times \mathbb{R} \times \mathbb{R}^{N} \rightarrow \mathbb{R}$ are supposed to be Carathéodory functions satisfying suitable structure conditions (see hypotheses (H1) and (H2) below) while $d \sigma$ denotes the usual $(N-1)$-dimensional surface measure and for $u \in V$ the generalized boundary values on $\partial \Omega$ are well defined in the sense of traces. For reasons of simplification we drop the notation for the trace operator.

The aim of our treatment is to present conditions on the set of constraints, namely $K$, such that every solution $u \in K$ of (1.1) is essentially bounded. In order to specify the space $V$ we will discuss two different cases: In Section 3 we choose $V=W^{1, p}(\Omega)$ with $1<p<\infty$ and in Section 4 we set $V=W^{1, p(\cdot)}(\Omega)$ with $p \in C(\bar{\Omega})$ and $1<\inf _{\bar{\Omega}} p$. These cases will be handled by different methods, the first one via Moser iteration and the second one via De Giorgi's iteration technique.

Due to the presence of the terms $j_{k}^{\circ}(x, s ; r)$, inequalities of type (1.1) are called variational-hemivariational inequalities which include several interesting problems as special cases.

2010 Mathematics Subject Classification. 35B45, 35J25, 35J60, 35J87.

Key words and phrases. a priori bounds, Clarke's gradient, De Giorgi iteration, generalized directional derivative, Moser iteration, variational-hemivariational inequality. 
a) If $K=V$ and $j_{k}(k=1,2)$ are smooth, problem (1.1) becomes

$$
\begin{aligned}
& \int_{\Omega} A(x, u, \nabla u) \cdot \nabla v d x+\int_{\Omega} F(x, u, \nabla u) v d x \\
& +\int_{\Omega} j_{1}^{\prime}(x, u) v d x+\int_{\partial \Omega} j_{2}^{\prime}(x, u) v d \sigma=0, \quad \forall v \in V,
\end{aligned}
$$

which means that $u \in V$ is a weak solution to the problem

$$
\begin{aligned}
-\operatorname{div} A(x, u, \nabla u)+F(x, u, \nabla u)+j_{1}^{\prime}(x, u) & =0 & & \text { in } \Omega, \\
A(x, u, \nabla u) \cdot \nu+j_{2}^{\prime}(x, u) & =0 & & \text { on } \partial \Omega,
\end{aligned}
$$

where $\nu(x)$ denotes the outer unit normal of $\Omega$ at $x \in \partial \Omega$. Regarding a priori bounds for such problems we refer to Winkert-Zacher [32, Theorem 1.1 and Corollary 1.2] (if $V=W^{1, p(\cdot)}(\Omega)$ ), Winkert [31, Theorem 4.1] (if $V=W^{1, p}(\Omega)$, see also Winkert [30, Proof of Proposition 5.2]) and Hu-Papageorgiou [18, Proposition 5] (if $V=W^{1, p}(\Omega)$ with homogeneous Neumann boundary condition).

b) If $j_{k}=0(k=1,2),(1.1)$ reduces to a classical variational inequality: Find $u \in K$ such that

$$
\int_{\Omega} A(x, u, \nabla u) \cdot \nabla(v-u) d x+\int_{\Omega} F(x, u, \nabla u)(v-u) d x \geq 0 \quad \forall v \in K .
$$

Boundedness results for solutions of such variational inequalities under suitable criteria on the set of constraints have been obtained by KovalevskyNicolosi [20, Theorem 2.8], where $V=W^{1, p}(\theta, \Omega)$ with $1<p<\infty$ and a positive weight $\theta$ satisfying $\theta \in L_{\mathrm{loc}}^{1}(\Omega)$ and $1 / \theta \in L_{\mathrm{loc}}^{1 /(p-1)}(\Omega)$ (see also Ježková [19] for local boundedness results). Concerning degenerated elliptic operators of high order we refer to a work of the same authors [21]. Recently, Gorban-Kovalevsky [16] have been studied the boundedness of solutions of degenerate anisotropic elliptic variational inequalities under certain conditions on the right-hand side and the set of constraints.

c) In case that $K$ is the whole space $V$ and $j_{k}(k=1,2)$ not necessarily smooth, problem (1.1) is a hemivariational inequality which contains as a special case the subsequent elliptic inclusion

$$
\begin{aligned}
-\operatorname{div} A(x, u, \nabla u)+F(x, u, \nabla u)+\partial j_{1}(x, u) \ni 0 & \text { in } \Omega, \\
A(x, u, \nabla u) \cdot \nu+\partial j_{2}(x, u) \ni 0 & \text { on } \partial \Omega,
\end{aligned}
$$

where the expression $\partial j_{k}(x, s)$ denotes the generalized gradient of the locally Lipschitz function $j_{k}(x, \cdot)$ in the sense of Clarke (see Section 2 for more details).

d) Let $V=W^{1, p}(\Omega)$ with $1<p<\infty$. If the operator $A$ satisfies appropriate monotonicity conditions with respect to the second and third argument and if the functions involved fulfill suitable structure conditions (similar to those in Section 3), then inequality (1.1) is equivalent to the multi-valued 
variational inequality: Find $u \in K$ such that

$$
\left\{\begin{array}{l}
\eta(x) \in \partial j_{1}(x, u(x)) \text { a.e. in } \Omega, \xi(x) \in \partial j_{2}(x, u(x)) \text { a.e. in } \partial \Omega \\
\int_{\Omega} A(x, u, \nabla u) \cdot \nabla(v-u) d x+\int_{\Omega} F(x, u, \nabla u)(v-u) d x \\
+\int_{\Omega} \eta(v-u) d x+\int_{\partial \Omega} \xi(v-u) d \sigma \geq 0, \quad \forall v \in K .
\end{array}\right.
$$

This result was published by Carl [5].

We point out that our results are more general than those in [20]. On the one hand we extend their results to variational-hemivariational inequalities including nonlinear boundary terms and on the other hand we have weaker assumptions on the set of constraints. Indeed, hypothesis 2.6(i) in [20] is not needed in our treatment. For the sake of convenience we do not use Sobolev spaces with weights, but this case can be done in the same way. Another novelty of this work is the treatment of variational-hemivariational inequalities restricted to sets $K$ belonging to variable exponent spaces $W^{1, p(\cdot)}(\Omega)$ with $p$ continuous on $\bar{\Omega}$. To the best of our knowledge, our a priori estimates have not been published before.

Notice that we do not suppose that the set $K$ is closed and convex in $V$. But in general, this is the typical assumption in the existence theory of inequalities like (1.1). Regarding existence and multiplicity results for problems of the form (1.1) we refer, without guarantee of completeness, to the papers of Carl [6], Kyritsi-Papageorgiou [23], Motreanu-Bonanno-Winkert [2], Bonanno-Winkert [3], Motreanu-Winkert [27] and the references therein. An overview about results to nonsmooth analysis and variational-hemivariational inequalities can be found in the monographs of Carl-Le-Motreanu [7] and Motreanu-Rădulescu [26]. We also point out a recent work of Carl [4] in which the class of variational-hemivariational inequalities has been extended to a more general class of inequalities.

The paper is organized as follows. In Section 2 we present some basic facts about nonsmooth analysis and the corresponding function spaces to problem (1.1). Furthermore, we prove an useful multiplicative inequality for boundary integrals. Section 3 handles the constant exponent case (i.e., $V=W^{1, p}(\Omega)$ with $1<p<\infty$ ) where we will apply Moser's iteration following the ideas of Drábek-Kufner-Nicolosi [12]. In the last section we extend our results to the variable exponent case (i.e. $V=W^{1, p(\cdot)}(\Omega)$ with $p \in C(\bar{\Omega})$ and $\left.1<\inf _{\bar{\Omega}} p\right)$ by applying De Giorgi's iteration. The results in this section are based on ideas of DiBenedetto [10], LadyženskajaSolonnikov-Ural'ceva [24], and Winkert-Zacher [32].

\section{Preliminaries AND hypotheses}

Let $\Omega$ be a bounded domain in $\mathbb{R}^{N}$ with Lipschitz boundary $\partial \Omega$ and let $p \in C(\bar{\Omega})$ with $p(x)>1$ for all $x \in \bar{\Omega}$. Setting $p^{-}:=\min _{x \in \bar{\Omega}} p(x)$ and $p^{+}:=\max _{x \in \bar{\Omega}} p(x)$, we have $p^{-}>1$ and $p^{+}<\infty$. The variable exponent Lebesgue space $L^{p(\cdot)}(\Omega)$ is defined by

$$
L^{p(\cdot)}(\Omega)=\left\{u \mid u: \Omega \rightarrow \mathbb{R} \text { is measurable and } \int_{\Omega}|u|^{p(x)} d x<+\infty\right\}
$$


equipped with the Luxemburg norm

$$
\|u\|_{L^{p(\cdot)}(\Omega)}=\inf \left\{\tau>0: \int_{\Omega}\left|\frac{u(x)}{\tau}\right|^{p(x)} d x \leq 1\right\} .
$$

By $W^{1, p(\cdot)}(\Omega)$ we identify the variable exponent Sobolev space which is defined by

$$
W^{1, p(\cdot)}(\Omega)=\left\{u \in L^{p(\cdot)}(\Omega):|\nabla u| \in L^{p(\cdot)}(\Omega)\right\}
$$

with the norm

$$
\|u\|_{W^{1, p(\cdot)}(\Omega)}=\|\nabla u\|_{L^{p(\cdot)}(\Omega)}+\|u\|_{L^{p(\cdot)}(\Omega)} .
$$

We refer to the papers of Fan-Zhao [15], Kováčik-Rákosník [22] and the monograph of Diening-Harjulehto-Hästö-Růžička [11] for more information and basic properties about variable exponent spaces. If $p(x) \equiv p$ is a constant, the usual Sobolev space $W^{1, p}(\Omega)$ is endowed with the norm

$$
\|u\|_{W^{1, p}(\Omega)}=\left(\int_{\Omega}|\nabla u|^{p} d x+\int_{\Omega}|u|^{p} d x\right)^{\frac{1}{p}} .
$$

Let us recall some basic facts on nonsmooth analysis. Let $(X,\|\cdot\|)$ be a real Banach space and denote by $X^{*}$ its dual space while the duality pairing between $X$ and $X^{*}$ is denoted by $\langle\cdot, \cdot\rangle$. The dual space $X^{*}$ is equipped with the dual norm $\|\cdot\|_{*}$, that is

$$
\|\xi\|_{*}=\sup \{\langle\xi, v\rangle: v \in X,\|v\| \leq 1\} .
$$

A function $j: X \rightarrow \mathbb{R}$ is said to be locally Lipschitz if for every $x \in X$ there exist a neighborhood $U$ of $x$ in $X$ and a constant $C>0$ such that

$$
|j(y)-j(z)| \leq C\|y-z\|, \quad \text { for all } y, z \in U .
$$

The generalized directional derivative of a locally Lipschitz function $j: X \rightarrow \mathbb{R}$ at a point $u \in X$ along the direction $v \in X$ is defined by

$$
j^{\circ}(u ; v):=\limsup _{x \rightarrow u, t \rightarrow 0^{+}} \frac{j(x+t v)-j(x)}{t} .
$$

Since $j$ is locally Lipschitz at $u$ we have $j^{\circ}(u ; v) \in \mathbb{R}$ for all $v \in X$. Furthermore, the function $j^{\circ}(u ; \cdot): X \rightarrow \mathbb{R}$ is subadditive, positively homogeneous and there holds the inequality

$$
\left|j^{\circ}(u ; v)\right| \leq C\|v\| \quad \text { for all } v \in X
$$

with $C$ being the Lipschitz constant of $j$ near the point $u \in X$.

The generalized gradient of a locally Lipschitz function $j: X \rightarrow \mathbb{R}$ at a point $u \in X$, denoted by $\partial j(u)$, is the subset of $X^{*}$ defined by

$$
\partial j(u):=\left\{\xi \in X^{*}: j^{\circ}(u ; v) \geq\langle\xi, v\rangle \text { for all } v \in X\right\},
$$

which is also known as Clarke's generalized gradient. Based on the Hahn-Banach theorem we easily verify that $\partial j(u)$ is nonempty. Moreover, $\partial j(u)$ is a convex, weak* compact subset of $X^{*}$ and it holds $\|\xi\|_{*} \leq C$ for all $\xi \in \partial j(u)$. For every $v \in X$, one has

$$
j^{\circ}(u ; v)=\max \{\langle\xi, v\rangle: \xi \in \partial j(u)\} .
$$


We refer to the monographs of Clarke [9] and Motreanu-Rădulescu [26] as well as the paper of Chang [8] for more details and properties of generalized directional derivatives and generalized gradients.

The next result is needed for the proof via Moser iteration to estimate the boundary integrals by suitable integrals defined in $\Omega$. The proof is based on appropriate embeddings and interpolation results of Besov and Sobolev Slobodeckij spaces.

Proposition 2.1. Let $\Omega \subset \mathbb{R}^{N}, N>1$, be a bounded domain with Lipschitz boundary $\partial \Omega$, let $1<p<\infty$, and let $q$ be such that $p \leq q<p_{*}$ with the critical exponent

$$
p_{*}= \begin{cases}\frac{(N-1) p}{N-p} & \text { if } p<N, \\ \infty & \text { if } p \geq N .\end{cases}
$$

Then, for every $\varepsilon>0$, there exist constants $a_{1}>0$ and $a_{2}>0$ such that

$$
\|u\|_{L^{q}(\partial \Omega)}^{p} \leq \varepsilon\|u\|_{W^{1, p}(\Omega)}^{p}+a_{1} \varepsilon^{-a_{2}}\|u\|_{L^{p}(\Omega)}^{p} \quad \text { for all } u \in W^{1, p}(\Omega) .
$$

Proof. Since $q<p_{*}$ we may fix a number $\theta \in(0,1)$ small enough such that

$$
q\left\{\begin{array}{ll}
\leq \frac{(N-1) p}{N-p+\theta p} & \text { if } p \leq N \\
<\frac{p-N}{\theta} & \text { if } p>N
\end{array} \text { and } \quad 1-\theta>\frac{1}{p} .\right.
$$

From Triebel $[28,3.3 .3]$, we have the continuous embedding

$$
B_{p, p}^{1-\theta}(\Omega)=W^{1-\theta, p}(\Omega) \rightarrow B_{p, p}^{1-\theta-\frac{1}{p}}(\partial \Omega)=W^{1-\theta-\frac{1}{p}, p}(\partial \Omega),
$$

where $B_{p, p}^{s}, s \in(0,1)$, denotes the Besov space which coincides with the Sobolev Slobodeckij space $W^{s, p}$. Note that the embedding (2.2) requires only a Lipschitz boundary as $1-\theta<1$.

From the choice of $\theta \in(0,1)$ and since $p \leq q$ (see also (2.1)) we get

$$
\left(1-\theta-\frac{1}{p}\right) p \begin{cases}<N-1 & \text { if } p \leq N \\ >N-1 & \text { if } p>N .\end{cases}
$$

Taking into account the Sobolev embedding theorem for fractional order Sobolev spaces (see Adams [1, Theorem 7.57]) gives

$$
W^{1-\theta-\frac{1}{p}, p}(\partial \Omega) \rightarrow L^{q}(\partial \Omega)
$$

for

$$
q \begin{cases}\leq \frac{(N-1) p}{N-1-\left(1-\theta-\frac{1}{p}\right) p}=\frac{(N-1) p}{N-p+\theta p} & \text { if }\left(1-\theta-\frac{1}{p}\right) p<N-1, \\ <\infty & \text { if }\left(1-\theta-\frac{1}{p}\right) p>N-1 .\end{cases}
$$

Actually, in case $\left(1-\theta-\frac{1}{p}\right) p>N-1$ we have the stronger embedding $W^{1-\theta-\frac{1}{p}, p}(\partial \Omega) \rightarrow$ $C(\partial \Omega)$.

Since $W^{1, p}(\Omega) \subset W^{1-\theta, p}(\Omega) \subset L^{p}(\Omega)$ are continuous embeddings we may apply real interpolation (see Triebel $[29,1.6 .2$ and 1.6.7])

$$
\left(L^{p}(\Omega), W^{1, p}(\Omega)\right)_{1-\theta, p}=W^{1-\theta, p}(\Omega),
$$

which implies the estimate

$$
\|u\|_{W^{1-\theta, p}(\Omega)} \leq \tilde{C}_{1}\|u\|_{W^{1, p}(\Omega)}^{1-\theta}\|u\|_{L^{p}(\Omega)}^{1-(1-\theta)} \quad \text { for all } u \in W^{1, p}(\Omega)
$$


with a positive constant $\tilde{C}_{1}$. Combining (2.2)-(2.4) and using Young's inequality with $\tilde{\delta}>0$ results in

$$
\begin{aligned}
\|u\|_{L^{q}(\partial \Omega)}^{p} & \leq \tilde{C}_{2} \tilde{\delta}^{1-\theta}\|u\|_{W^{1, p}(\Omega)}^{(1-\theta) p} \tilde{\delta}^{-1+\theta}\|u\|_{L^{p}(\Omega)}^{\theta p} \\
& \leq \tilde{C}_{2}\left(\tilde{\delta}\|u\|_{W^{1, p}(\Omega)}^{p}+\tilde{\delta}^{\frac{-1+\theta}{\theta}}\|u\|_{L^{p}(\Omega)}^{p}\right) .
\end{aligned}
$$

Setting $\tilde{\delta}:=\frac{\varepsilon}{\widetilde{C_{2}}}$ with arbitrary $\varepsilon>0$ provides the desired estimate.

Finally, we conclude this section by fixing our notation. If $s \in \mathbb{R}$ we write $s_{+}=\max (s, 0)$ and $s_{-}=\min (s, 0)$. For functions $u, v: \Omega \rightarrow \mathbb{R}$ we use the notation $u \wedge v=\min (u, v), u \vee v=\max (u, v), K \wedge K=\{u \wedge v: u, v \in K\}$, and $K \vee K=\{u \vee v: u, v \in K\}$. If the set $K \subseteq V$ satisfies

$$
K \wedge K \subset K \text { and } K \vee K \subset K,
$$

we say that $K$ has lattice structure. Note that $V$ has lattice structure, that means,

$$
V \wedge V \subset V \text { and } V \vee V \subset V
$$

(see Heinonen-Kilpeläinen-Martio [17, Theorem 1.20] if $V=W^{1, p}(\Omega)$ and Le [25, Lemma 3.2] if $\left.V=W^{1, p(\cdot)}(\Omega)\right)$. Throughout the paper we will denote by $M_{i}$ and $\hat{M}_{j}, i, j=1,2, \ldots$ positive constants depending on the given data and the Lebesgue measure on $\mathbb{R}^{N}$ is given by $|\cdot|_{N}$.

\section{The Case $V=W^{1, p}(\Omega)$ Via Moser iteration}

We start our treatment with the constant exponent case and use Moser's iteration to prove $L^{\infty}$-bounds for solutions of inequality (1.1). In this section we suppose the following assumptions.

(H1) The mappings $A: \Omega \times \mathbb{R} \times \mathbb{R}^{N} \rightarrow \mathbb{R}^{N}$ and $F: \Omega \times \mathbb{R} \times \mathbb{R}^{N} \rightarrow \mathbb{R}$ are supposed to satisfy a Carathéodory condition while $x \mapsto j_{1}(x, s), x \mapsto j_{2}(x, s)$ are measurable for all $s \in \mathbb{R}$ and $s \mapsto j_{1}(x, s), s \mapsto j_{2}(x, s)$ are locally Lipschitz for a.a. $x \in \Omega$ and for a.a. $x \in \partial \Omega$, respectively. In addition the subsequent structure conditions are assumed:

(i) $A(x, s, \xi) \cdot \xi \geq a_{1}|\xi|^{p}-a_{2}|s|^{q_{1}}-a_{3} \quad$ for a.a. $x \in \Omega$;

(ii) $|A(x, s, \xi)| \leq a_{4}|\xi|^{p-1}+a_{5}|s|^{q_{1} \frac{p-1}{p}}+a_{6} \quad$ for a.a. $x \in \Omega$;

(iii) $|F(x, s, \xi)| \leq b_{1}|\xi|^{\frac{q_{1}-1}{q_{1}}}+b_{2}|s|^{q_{1}-1}+b_{3} \quad$ for a.a. $x \in \Omega$;

(iv) $|\eta| \leq c_{1}|s|^{q_{1}-1}+c_{2} \quad$ for a.a. $x \in \Omega$, for all $\eta \in \partial j_{1}(x, s)$;

(v) $|\tau| \leq d_{1}|s|^{q_{2}-1}+d_{2}, \quad$ for a.a. $x \in \partial \Omega$, for all $\tau \in \partial j_{2}(x, s)$;

and for all $s \in \mathbb{R}$, and all $\xi \in \mathbb{R}^{N}$ with positive constants $a_{i}, b_{j}, c_{k}, d_{l}$ $(i \in\{1, \ldots, 6\}, j \in\{1, \ldots, 3\}, k, l \in\{1,2\})$ and fixed numbers $p, q_{1}, q_{2}$ such that

$$
1<p<\infty, \quad p \leq q_{1}<p^{*}, \quad p \leq q_{2}<p_{*}
$$

with the critical exponents

$$
p^{*}=\left\{\begin{array}{ll}
\frac{N p}{N-p} & \text { if } p<N, \\
+\infty & \text { if } p \geq N,
\end{array} \quad p_{*}= \begin{cases}\frac{(N-1) p}{N-p} & \text { if } p<N \\
+\infty & \text { if } p \geq N\end{cases}\right.
$$


Recalling

$$
\partial j_{k}(x, s)=\left\{\xi \in \mathbb{R}: j_{k}^{\circ}(x, s ; r) \geq \xi r, \forall r \in \mathbb{R}\right\}
$$

for a.a. $x \in \Omega(k=1)$, respectively, for a.a. $x \in \partial \Omega(k=2)$ we easily derive the estimates

$$
\begin{array}{ll}
\left|j_{1}^{\circ}(x, s ; r)\right| \leq\left(c_{1}|s|^{q_{1}-1}+c_{2}\right)|r| & \text { for a.a. } x \in \Omega \text { and for all } s, r \in \mathbb{R}, \\
\left|j_{2}^{\circ}(x, s ; r)\right| \leq\left(d_{1}|s|^{q_{2}-1}+d_{2}\right)|r| & \text { for a.a. } x \in \partial \Omega \text { and for all } s, r \in \mathbb{R},
\end{array}
$$

thanks to (H1)(iv) and (v). From the embeddings $W^{1, p}(\Omega) \rightarrow L^{q_{1}}(\Omega)$ and $W^{1, p}(\Omega) \rightarrow$ $L^{q_{2}}(\partial \Omega)$ (see Adams [1]) and the growth conditions in (H1)(ii), (iii), (3.1) as well as (3.2) we see that the integrals in (1.1) are finite.

For $u \in W^{1, p}(\Omega)$ and $\alpha, \beta>0$ we define the functions $v_{\alpha}(x):=\min \left(u_{+}(x), \alpha\right)$ and $v_{\beta}(x):=\max \left(u_{-}(x),-\beta\right)$ which belong both to $W^{1, p}(\Omega)$. We suppose the following conditions on the set $K$.

(K1) For $u \in K, \alpha>0$, and $\kappa>0$ there exists $t>0$ such that

$$
\varphi=u-t v_{\alpha}^{\kappa p} u \in K \text {. }
$$

(K2) For $u \in K, \beta>0$, and $\kappa>0$ there exists $h>0$ such that

$$
\psi=u-h\left(-v_{\beta}\right)^{\kappa p} u \in K
$$

Note that both $\varphi$ and $\psi$ are elements of $W^{1, p}(\Omega)$ and their gradients are given by

$$
\nabla \varphi=\nabla u-t \kappa p v_{\alpha}^{\kappa p-1} \nabla v_{\alpha} u-t v_{\alpha}^{\kappa p} \nabla u=\nabla u-t \kappa p v_{\alpha}^{\kappa p} \nabla v_{\alpha}-t v_{\alpha}^{\kappa p} \nabla u
$$

respectively,

$$
\begin{aligned}
\nabla \psi & =\nabla u-h \kappa p\left(-v_{\beta}\right)^{\kappa p-1} \nabla\left(-v_{\beta}\right) u-h\left(-v_{\beta}\right)^{\kappa p} \nabla u \\
& =\nabla u+h \kappa p\left(-v_{\beta}\right)^{\kappa p} \nabla\left(-v_{\beta}\right)-h\left(-v_{\beta}\right)^{\kappa p} \nabla u .
\end{aligned}
$$

We have the following result.

Theorem 3.1. Let hypothesis (H1) be satisfied and let $u \in K$ be a solution of (1.1). Then there exists a constant $C_{1}>0$ such that the following assertions hold.

(1) If condition (K1) is satisfied, then

$$
\underset{x \in \Omega}{\operatorname{ess} \sup } u(x) \leq C_{1} \text {. }
$$

(2) If condition (K2) is satisfied, then

$$
\underset{x \in \Omega}{\operatorname{ess} \inf } u(x) \geq-C_{1} .
$$

Proof. We start with (1) and assume, without loss of generality, that $p<q_{1}$ and $p<q_{2}$. The cases $p=q_{1}$ and/or $p=q_{2}$ work similarly. Thanks to (K1) we may take $v=\varphi=u-t v_{\alpha}^{\kappa p} u \in K$ in (1.1). This gives

$$
\begin{aligned}
& t \kappa p \int_{\Omega} A(x, u, \nabla u) \cdot \nabla v_{\alpha} v_{\alpha}^{\kappa p} d x+t \int_{\Omega} A(x, u, \nabla u) \cdot \nabla u v_{\alpha}^{\kappa p} d x \\
& \leq-t \int_{\Omega} F(x, u, \nabla u) v_{\alpha}^{\kappa p} u d x+\int_{\Omega} j_{1}^{\circ}\left(x, u ;-t v_{\alpha}^{\kappa p} u\right) d x \\
& \quad+\int_{\partial \Omega} j_{2}^{\circ}\left(x, u ;-t v_{\alpha}^{\kappa p} u\right) d \sigma .
\end{aligned}
$$

Since $j_{k}(k=1,2)$ are positively homogeneous with respect to the third argument (cf. [9, Proposition 2.1.1]), we can divide inequality (3.3) by $t>0$. 
Let us estimate the several terms in (3.3). We start with the left-hand side of (3.3) by applying hypothesis (H1)(i) and Hölder's inequality. We obtain

$$
\begin{aligned}
& \kappa p \int_{\Omega} A(x, u, \nabla u) \cdot \nabla v_{\alpha} v_{\alpha}^{\kappa p} d x+\int_{\Omega} A(x, u, \nabla u) \cdot \nabla u v_{\alpha}^{\kappa p} d x \\
& =\kappa p \int_{\Omega} A\left(x, v_{\alpha}, \nabla v_{\alpha}\right) \cdot \nabla v_{\alpha} v_{\alpha}^{\kappa p} d x+\int_{\Omega} A\left(x, u_{+}, \nabla u_{+}\right) \cdot \nabla u_{+} v_{\alpha}^{\kappa p} d x \\
& \geq \kappa p \int_{\Omega}\left(a_{1}\left|\nabla v_{\alpha}\right|^{p}-a_{2}\left|v_{\alpha}\right|^{q_{1}}-a_{3}\right) v_{\alpha}^{\kappa p} d x \\
& \quad+\int_{\Omega}\left(a_{1}\left|\nabla u_{+}\right|^{p}-a_{2}\left|u_{+}\right|^{q_{1}}-a_{3}\right) v_{\alpha}^{\kappa p} d x \\
& \geq \kappa p\left(a_{1} \int_{\Omega}\left|\nabla v_{\alpha}\right|^{p} v_{\alpha}^{\kappa p} d x-a_{2} \int_{\Omega} u_{+}^{(\kappa+1) p} u_{+}^{q_{1}-p} d x-a_{3} \int_{\Omega} u_{+}^{\kappa p} d x\right) \\
& \quad+a_{1} \int_{\Omega}\left|\nabla u_{+}\right|^{p} v_{\alpha}^{\kappa p} d x-\left(a_{2}+a_{3}\right) \int_{\Omega} u_{+}^{(\kappa+1) p} u_{+}^{q_{1}-p} d x-a_{3}|\Omega|_{N} \\
& \geq a_{1} \kappa p \int_{\Omega}\left|\nabla v_{\alpha}\right|^{p} v_{\alpha}^{\kappa p} d x+a_{1} \int_{\Omega}\left|\nabla u_{+}\right|^{p} v_{\alpha}^{\kappa p} d x \\
& \quad-M_{1}(\kappa p+1)\left\|u_{+}^{\kappa+1}\right\|_{L^{q_{1}}(\Omega)}^{p}-M_{2} .
\end{aligned}
$$

By means of (H1)(iii) combined with Hölder's inequality and Young's inequality with $\varepsilon_{1}>0$ the first term on the right-hand side can be estimated through

$$
\begin{aligned}
& -\int_{\Omega} F(x, u, \nabla u) v_{\alpha}^{\kappa p} u d x \\
& \leq b_{1} \int_{\Omega} \varepsilon_{1}^{\frac{q_{1}-1}{q_{1}}}\left|\nabla u_{+}\right|^{p \frac{q_{1}-1}{q_{1}}} u_{+}^{\kappa p \frac{q_{1}-1}{q_{1}}} \varepsilon_{1}^{-\frac{q_{1}-1}{q_{1}}} u_{+}^{\kappa p\left(1-\frac{q_{1}-1}{q_{1}}\right)+1} d x \\
& \quad+\left(b_{2}+b_{3}\right) \int_{\Omega} u_{+}^{(\kappa+1) p} u_{+}^{q_{1}-p} d x+b_{3}|\Omega|_{N} \\
& \leq \frac{\varepsilon_{1} b_{1}}{(\kappa+1)^{p}} \int_{\Omega}\left|\nabla u_{+}^{\kappa+1}\right|^{p} d x+M_{3}\left(\varepsilon_{1}^{-\left(q_{1}-1\right)}+1\right)\left\|u_{+}^{\kappa+1}\right\|_{L^{q_{1}(\Omega)}}^{p}+M_{4} .
\end{aligned}
$$

Owing to (3.1) combined with Hölder's inequality the second integral on the righthand side of (3.3) gives

$$
\begin{aligned}
\int_{\Omega} j_{1}^{\circ}\left(x, u ;-v_{\alpha}^{\kappa p} u\right) d x & \leq \int_{\Omega}\left(c_{1}|u|^{q_{1}-1}+c_{2}\right) u_{+}^{\kappa p+1} d x \\
& \leq M_{5}\left\|u_{+}^{\kappa+1}\right\|_{L^{q_{1}}(\Omega)}^{p}+M_{6} .
\end{aligned}
$$

In the same way, using (3.2) and again Hölder's inequality, we get for the last integral

$$
\begin{aligned}
\int_{\partial \Omega} j_{2}^{\circ}\left(x, u ;-v_{\alpha}^{\kappa p} u\right) d \sigma & \leq \int_{\partial \Omega}\left(d_{1}|u|^{q_{2}-1}+d_{2}\right) u_{+}^{\kappa p+1} d \sigma \\
& \leq M_{7}\left\|u_{+}^{\kappa+1}\right\|_{L^{q_{2}(\partial \Omega)}}^{p}+M_{8} .
\end{aligned}
$$


Now, combining (3.3)-(3.7) we have

$$
\begin{aligned}
& a_{1} \kappa p \int_{\Omega}\left|\nabla v_{\alpha}\right|^{p} v_{\alpha}^{\kappa p} d x+a_{1} \int_{\Omega}\left|\nabla u_{+}\right|^{p} v_{\alpha}^{\kappa p} d x \\
& \leq \frac{\varepsilon_{1} b_{1}}{(\kappa+1)^{p}} \int_{\Omega}\left|\nabla u_{+}^{\kappa+1}\right|^{p} d x+M_{9}\left(\varepsilon_{1}^{-\left(q_{1}-1\right)}+1\right)\left\|u_{+}^{\kappa+1}\right\|_{L^{q_{1}(\Omega)}}^{p} \\
& \quad+M_{10}\left\|u_{+}^{\kappa+1}\right\|_{L^{q_{2}(\partial \Omega)}}^{p}+M_{11} .
\end{aligned}
$$

Taking into account Fatou's lemma yields

$$
\begin{aligned}
& a_{1} \frac{\kappa p+1}{(\kappa+1)^{p}} \int_{\Omega}\left|\nabla u_{+}^{\kappa+1}\right|^{p} d x \\
& \leq \frac{\varepsilon_{1} b_{1}}{(\kappa+1)^{p}} \int_{\Omega}\left|\nabla u_{+}^{\kappa+1}\right|^{p} d x+M_{9}\left(\varepsilon_{1}^{-\left(q_{1}-1\right)}+1\right)\left\|u_{+}^{\kappa+1}\right\|_{L^{q_{1}(\Omega)}}^{p} \\
& \quad \quad+M_{10}\left\|u_{+}^{\kappa+1}\right\|_{L^{q_{2}(\partial \Omega)}}^{p}+M_{11} .
\end{aligned}
$$

Choosing $\varepsilon_{1}=\frac{a_{1}}{2 b_{1}}(\kappa p+1)$ implies $\varepsilon_{1}^{-\left(q_{1}-1\right)} \leq M_{12}$. Then, (3.8) becomes

$$
\begin{aligned}
& \frac{a_{1}}{2} \frac{\kappa p+1}{(\kappa+1)^{p}} \int_{\Omega}\left|\nabla u_{+}^{\kappa+1}\right|^{p} d x \\
& \leq M_{13}\left\|u_{+}^{\kappa+1}\right\|_{L^{q_{1}(\Omega)}}^{p}+M_{10}\left\|u_{+}^{\kappa+1}\right\|_{L^{q_{2}(\partial \Omega)}}^{p}+M_{11} .
\end{aligned}
$$

Now, we may apply Proposition 2.1 to the boundary term in (3.9) (with $q=q_{2}$, $\left.\varepsilon=\varepsilon_{2}\right)$ and Hölder's inequality as before to get

$$
\begin{aligned}
& \left\|u_{+}^{\kappa+1}\right\|_{L^{q_{2}(\partial \Omega)}}^{p} \\
& \leq \varepsilon_{2} \int_{\Omega}\left|\nabla u_{+}^{\kappa+1}\right|^{p} d x+\varepsilon_{2} \int_{\Omega} u_{+}^{(\kappa+1) p} d x+a_{1} \varepsilon_{2}^{-a_{2}} \int_{\Omega} u_{+}^{(\kappa+1) p} d x \\
& \leq \varepsilon_{2} \int_{\Omega}\left|\nabla u_{+}^{\kappa+1}\right|^{p} d x+M_{14}\left(\varepsilon_{2}+\varepsilon_{2}^{-a_{2}}\right)\left\|u_{+}^{\kappa+1}\right\|_{L^{q_{1}}(\Omega)}^{p} .
\end{aligned}
$$

Combining (3.9) and (3.10) results in

$$
\begin{aligned}
& \left(\frac{a_{1}}{2} \frac{\kappa p+1}{(\kappa+1)^{p}}-M_{10} \varepsilon_{2}\right) \int_{\Omega}\left|\nabla u_{+}^{\kappa+1}\right|^{p} d x \\
& \leq M_{15}\left(\varepsilon_{2}+\varepsilon_{2}^{-a_{2}}+1\right)\left\|u_{+}^{\kappa+1}\right\|_{L^{q_{1}(\Omega)}}^{p}+M_{11} .
\end{aligned}
$$

The choice $\varepsilon_{2}=\frac{a_{1}}{4 M_{10}} \frac{\kappa p+1}{(\kappa+1)^{p}}$ gives

$$
\frac{a_{1}}{4} \frac{\kappa p+1}{(\kappa+1)^{p}} \int_{\Omega}\left|\nabla u_{+}^{\kappa+1}\right|^{p} d x \leq M_{16}\left(\frac{(\kappa+1)^{p}}{\kappa p+1}\right)^{M_{17}}\left\|u_{+}^{\kappa+1}\right\|_{L^{q_{1}(\Omega)}}^{p}+M_{11} .
$$

Finally, we divide (3.11) by $\frac{a_{1}}{4} \frac{\kappa p+1}{(\kappa+1)^{p}}>0$ and add on both sides the integral $\int_{\Omega} u_{+}^{(\kappa+1) p} d x$ which leads to

$$
\begin{aligned}
& \int_{\Omega}\left|\nabla u_{+}^{\kappa+1}\right|^{p} d x+\int_{\Omega} u_{+}^{(\kappa+1) p} d x \\
& \leq M_{18}\left(\frac{(\kappa+1)^{p}}{\kappa p+1}\right)^{M_{19}}\left(\left\|u_{+}^{\kappa+1}\right\|_{L^{q_{1}(\Omega)}}^{p}+1\right),
\end{aligned}
$$

where Hölder's inequality was again taken into account on the right-hand side. 
Having regard to the continuous embedding $W^{1, p}(\Omega) \rightarrow L^{\tilde{p}^{*}}(\Omega)$ with

$$
\tilde{p}^{*}= \begin{cases}\frac{N p}{N-p} & \text { if } p<N \\ 2 q_{1} & \text { if } p \geq N\end{cases}
$$

we obtain

$$
\begin{aligned}
\left\|u_{+}\right\|_{L^{(\kappa+1) \tilde{p}^{*}}(\Omega)} & =\left\|u_{+}^{\kappa+1}\right\|_{L^{\tilde{p}^{*}}(\Omega)}^{\frac{1}{\kappa+1}} \\
& \leq M_{20}^{\frac{1}{\kappa+1}}\left\|u_{+}^{\kappa+1}\right\|_{W^{1, p}(\Omega)}^{\frac{1}{\kappa+1}} \\
& =M_{20}^{\frac{1}{\kappa+1}}\left(\int_{\Omega}\left|\nabla u_{+}^{\kappa+1}\right|^{p} d x+\int_{\Omega} u_{+}^{(\kappa+1) p} d x\right)^{\frac{1}{(\kappa+1) p}},
\end{aligned}
$$

where $M_{20}>0$ denotes the embedding constant. Combining (3.12) and (3.13) we derive

$$
\begin{aligned}
& \left\|u_{+}\right\|_{L^{(\kappa+1) \tilde{p}^{*}}(\Omega)} \\
& \leq M_{20}^{\frac{1}{\kappa+1}}\left(\int_{\Omega}\left|\nabla u_{+}^{\kappa+1}\right|^{p} d x+\int_{\Omega} u_{+}^{(\kappa+1) p} d x\right)^{\frac{1}{(\kappa+1) p}} \\
& \leq M_{20}^{\frac{1}{\kappa+1}} M_{18}^{\frac{1}{(\kappa+1) p}}\left(\frac{(\kappa+1)^{M_{19}}}{(\kappa p+1)^{\frac{M_{19}}{p}}}\right)^{\frac{1}{\kappa+1}}\left(\left\|u_{+}^{\kappa+1}\right\|_{L^{q_{1}}(\Omega)}^{p}+1\right)^{\frac{1}{(\kappa+1) p}} .
\end{aligned}
$$

Observe that

$$
\left(\frac{(\kappa+1)^{M_{19}}}{(\kappa p+1)^{\frac{M_{19}}{p}}}\right)^{\frac{1}{\sqrt{\kappa+1}}} \geq 1 \quad \text { and } \quad \lim _{\kappa \rightarrow \infty}\left(\frac{(\kappa+1)^{M_{19}}}{(\kappa p+1)^{\frac{M_{19}}{p}}}\right)^{\frac{1}{\sqrt{\kappa+1}}}=1 .
$$

Hence, we find a constant $M_{21}>1$ such that

$$
\left(\frac{(\kappa+1)^{M_{19}}}{(\kappa p+1)^{\frac{M_{19}}{p}}}\right)^{\frac{1}{\kappa+1}} \leq M_{21}^{\frac{1}{\sqrt{\kappa+1}}}
$$

Applying (3.15) to (3.14) gives the estimate

$$
\left\|u_{+}\right\|_{L^{(\kappa+1) \tilde{p}^{*}}(\Omega)} \leq M_{20}^{\frac{1}{\kappa+1}} M_{18}^{\frac{1}{(\kappa+1) p}} M_{21}^{\frac{1}{\sqrt{\kappa+1}}}\left(\left\|u_{+}^{\kappa+1}\right\|_{L^{q_{1}}(\Omega)}^{p}+1\right)^{\frac{1}{(\kappa+1) p}}
$$

Now we can start with the typical bootstrap arguments. Choosing $\kappa$ such that

$$
\begin{aligned}
& \kappa_{1}:\left(\kappa_{1}+1\right) q_{1}=\tilde{p}^{*}, \\
& \kappa_{2}:\left(\kappa_{2}+1\right) q_{1}=\left(\kappa_{1}+1\right) \tilde{p}^{*}, \\
& \kappa_{3}:\left(\kappa_{3}+1\right) q_{1}=\left(\kappa_{2}+1\right) \tilde{p}^{*},
\end{aligned}
$$

we see that

$$
\left\|u_{+}\right\|_{L^{(\kappa+1) \tilde{p}^{*}(\Omega)}} \leq C(\kappa)
$$

for any finite number $\kappa$, where $C(\kappa)$ is a positive constant depending on $\kappa$. Thus, $u_{+} \in L^{r}(\Omega)$ for any $r \in(1, \infty)$. 
In order to prove the uniform estimate with respect to $\kappa$ we argue as follows. If there is a sequence $\kappa_{n} \rightarrow \infty$ such that

$$
\int_{\Omega} u_{+}^{\left(\kappa_{n}+1\right) q_{1}} d x \leq 1
$$

we directly obtain

$$
\left\|u_{+}\right\|_{L^{\infty}(\Omega)} \leq 1
$$

In the opposite case there exists a number $\kappa_{0}>0$ such that

$$
\int_{\Omega} u_{+}^{(\kappa+1) q_{1}} d x>1 \quad \text { for any } \kappa \geq \kappa_{0} .
$$

Then we conclude from (3.16)

$$
\begin{aligned}
\left\|u_{+}\right\|_{L^{(\kappa+1) \tilde{p}^{*}}(\Omega)} & \leq M_{20}^{\frac{1}{\kappa+1}} M_{18}^{\frac{1}{\kappa+1) p}} M_{21}^{\frac{1}{\sqrt{\kappa+1}}}\left(2\left\|u_{+}^{\kappa+1}\right\|_{L^{q_{1}}(\Omega)}^{p}\right)^{\frac{1}{(\kappa+1) p}} \\
& \leq M_{20}^{\frac{1}{\kappa+1}} M_{22}^{\frac{1}{\kappa+1) p}} M_{21}^{\frac{1}{\sqrt{\kappa+1}}}\left\|u_{+}\right\|_{L^{(\kappa+1) q_{1}}(\Omega)} .
\end{aligned}
$$

Applying again the bootstrap arguments we define a sequence $\left(\kappa_{n}\right)$ such that

$$
\begin{aligned}
& \kappa_{1}:\left(\kappa_{1}+1\right) q_{1}=\left(\kappa_{0}+1\right) \tilde{p}^{*}, \\
& \kappa_{2}:\left(\kappa_{2}+1\right) q_{1}=\left(\kappa_{1}+1\right) \tilde{p}^{*}, \\
& \kappa_{3}:\left(\kappa_{3}+1\right) q_{1}=\left(\kappa_{2}+1\right) \tilde{p}^{*},
\end{aligned}
$$

By induction, from (3.17) and (3.18), we obtain

$$
\begin{aligned}
\left\|u_{+}\right\|_{L^{\left(\kappa_{n}+1\right) \tilde{p}^{*}}(\Omega)} & \leq M_{20}^{\frac{1}{\kappa_{n}+1}} M_{22}^{\frac{1}{\left(\kappa_{n}+1\right) p}} M_{21}^{\frac{1}{\sqrt{\kappa_{n}+1}}}\left\|u_{+}\right\|_{L^{\left(\kappa_{n}+1\right) q_{1}}(\Omega)} \\
& =M_{20}^{\frac{1}{\kappa_{n}+1}} M_{22}^{\frac{1}{\left(\kappa_{n}+1\right) p}} M_{21}^{\frac{1}{\sqrt{\kappa_{n}+1}}}\left\|u_{+}\right\|_{L^{\left(\kappa_{n-1}+1\right) \tilde{p}^{*}}(\Omega)}
\end{aligned}
$$

for any $n \in \mathbb{N}$, where the sequence $\left(\kappa_{n}\right)$ is chosen such that $\left(\kappa_{n}+1\right)=\left(\kappa_{0}+1\right)\left(\frac{\tilde{p}^{*}}{q_{1}}\right)^{n}$. Then, we have

$$
\left\|u_{+}\right\|_{L^{\left(\kappa_{n}+1\right) \tilde{p}^{*}}(\Omega)} \leq M_{20}^{\sum_{i=1}^{n} \frac{1}{\kappa_{i}+1}} M_{22}^{\sum_{i=1}^{n} \frac{1}{\left(\kappa_{i}+1\right) p}} M_{21}^{\sum_{i=1}^{n} \frac{1}{\sqrt{\kappa_{i}+1}}}\left\|u_{+}\right\|_{L^{\left(\kappa_{0}+1\right) \tilde{p}^{*}}(\Omega)},
$$

with $\left(\kappa_{n}+1\right) \tilde{p}^{*} \rightarrow \infty$ as $n \rightarrow \infty$. Since $\frac{1}{\kappa_{i}+1}=\frac{1}{\kappa_{0}+1}\left(\frac{q_{1}}{\tilde{p}^{*}}\right)^{i}$ and $\frac{q_{1}}{\tilde{p}^{*}}<1$ there is a constant $M_{23}>0$ such that

$$
\left\|u_{+}\right\|_{L^{\left(\kappa_{n}+1\right) \tilde{p}^{*}(\Omega)}} \leq M_{23}\left\|u_{+}\right\|_{L^{\left(k_{0}+1\right) \tilde{p}^{*}}(\Omega)} .
$$

Since $u_{+} \in L^{r}(\Omega)$ for any $r \in(1, \infty)$, the right-hand side of (3.19) is finite. By means of (3.19) it follows that

$$
\underset{x \in \Omega}{\operatorname{ess} \sup } u_{+}(x) \leq C_{1}
$$

(see Drábek-Kufner-Nicolosi [12, proof of Lemma 3.2]). 
The second part can be proved similarly. We take $v=\psi=u-h\left(-v_{\beta}\right)^{\kappa p} u$ in (1.1) which leads to

$$
\begin{aligned}
& h \kappa p \int_{\Omega} A(x, u, \nabla u) \cdot \nabla v_{\beta}\left(-v_{\beta}\right)^{\kappa p} d x+h \int_{\Omega} A(x, u, \nabla u) \cdot \nabla u\left(-v_{\beta}\right)^{\kappa p} d x \\
& \leq-h \int_{\Omega} F(x, u, \nabla u)\left(-v_{\beta}\right)^{\kappa p} u d x+\int_{\Omega} j_{1}^{\circ}\left(x, u ;-h\left(-v_{\beta}\right)^{\kappa p} u\right) d x \\
& \quad+\int_{\partial \Omega} j_{2}^{\circ}\left(x, u ;-h\left(-v_{\beta}\right)^{\kappa p} u\right) d \sigma .
\end{aligned}
$$

Applying again the structure conditions in (H1) combined with Hölder's and Young's inequality to (3.20) the statement in (2) can be shown as in the first part. This completes the proof.

As a direct consequence of Theorem 3.1 we obtain the following corollary.

Corollary 3.2. Assume hypotheses (H1), (K1), (K2) and let $u \in K$ be a solution of (1.1). Then there holds

$$
\underset{x \in \Omega}{\operatorname{ess} \sup }|u(x)| \leq C_{1},
$$

where the constant $C_{1}$ is the same as in Theorem 3.1.

Remark 3.3. Note that the constant $C_{1}$ in Theorem 3.1 depends on the given data and on the solution $u \in K$, that is,

$$
C_{1}=C_{1}\left(p, q_{1}, q_{2}, a_{1}, a_{2}, a_{3}, b_{1}, b_{2}, b_{3}, c_{1}, c_{2}, d_{1}, d_{2}, \Omega, u\right) .
$$

The assumption (H1)(ii) is not needed in the proof of Theorem 3.1. It should be noted that the finiteness of the integrals

$$
\int_{\Omega}\left|\nabla u_{+}^{\kappa+1}\right|^{p} d x, \quad \int_{\Omega} u_{+}^{(\kappa+1) q_{1}} d x
$$

is shown in the end of the proof of Theorem 3.1 by a suitable choice of the parameter $\kappa$. This is a typical proceeding in the usage of the Moser iteration.

Remark 3.4. If $V=W_{0}^{1, p}(\Omega)$ being the usual Sobolev space consisting of the elements of $W^{1, p}(\Omega)$ with zero traces on $\partial \Omega$, problem (1.1) reduces to

$$
\begin{gathered}
u \in K: \quad \int_{\Omega} A(x, u, \nabla u) \cdot \nabla(v-u) d x+\int_{\Omega} F(x, u, \nabla u)(v-u) d x \\
+\int_{\Omega} j_{1}^{\circ}(x, u ; v-u) d x \geq 0
\end{gathered}
$$

for all $v \in K \subseteq W_{0}^{1, p}(\Omega)$. We observe that the results ensured by Theorem 3.1 still hold for problems of type (3.21). In this case we do not need Proposition 2.1 and the proof becomes more simple. In general, the assumptions in (K1) and (K2) are satisfied if $K=W_{0}^{1, p}(\Omega)$ and $K=W^{1, p}(\Omega)$.

Remark 3.5. As already mentioned in the Introduction our assumptions on the set of constraints are weaker than those in Kovalevsky-Nikolosi [20]. Precisely, Hypothesis 2.6(i) in [20] is not necessary in our proof.

Let us consider some examples of suitable sets $K$. To this end, let $\vartheta, \vartheta_{j}, \omega, \psi$ : $\Omega \rightarrow \mathbb{R}, j=1, \ldots, N$, be given functions such that $\vartheta \geq 0, \omega \leq 0 \leq \psi$ a.e. in $\Omega$. 
ON THE BOUNDEDNESS OF SOLUTIONS TO ELLIPTIC VARIATIONAL INEQUALITIES 13

Example 3.6. Let $\Theta: \Omega \times \mathbb{R} \rightarrow \mathbb{R}$ be a function such that $s \rightarrow \Theta(x, s)$ is convex for a.a. $x \in \Omega$ and such that $\Theta(x, 0) \leq 0$ for a.a. $x \in \Omega$. Then the set

$$
K=\left\{u \in W^{1, p}(\Omega): \Theta(x, u(x)) \leq 0 \quad \text { a.e. in } \Omega\right\}
$$

fulfills the conditions (K1), (K2) with $t=\alpha^{-\kappa p}$ and $h=\beta^{-\kappa p}$. In particular, $\Theta$ can be of the following form

$$
\Theta(x, s)=s-\vartheta(x), \quad \Theta(x, s)=-s-\vartheta(x), \quad \Theta(x, s)=(s-\omega(x))(s-\psi(x)) .
$$

From this choice we see that the subsequent sets satisfy our assumptions:

$$
\begin{aligned}
& K=\left\{u \in W^{1, p}(\Omega): u \leq \vartheta \quad \text { a.e. in } \Omega\right\}, \\
& K=\left\{u \in W^{1, p}(\Omega): u \geq-\vartheta \quad \text { a.e. in } \Omega\right\}, \\
& K=\left\{u \in W^{1, p}(\Omega): \omega \leq u \leq \psi \quad \text { a.e. in } \Omega\right\} .
\end{aligned}
$$

Example 3.7. Let $\Upsilon: \Omega \times \mathbb{R}^{N} \rightarrow \mathbb{R}$ be a function such that $\xi \rightarrow \Upsilon(x, \xi)$ is convex for a.a. $x \in \Omega$ and such that $\Upsilon(x, 0) \leq 0$ for a.a. $x \in \Omega$. Then the set

$$
K=\left\{u \in W^{1, p}(\Omega): \Upsilon(x, \nabla u(x)) \leq 0 \quad \text { a.e. in } \Omega\right\}
$$

fulfills the conditions (K1), (K2) with $t=(\kappa p+1)^{-1} \alpha^{-\kappa p}$ and $h=(\kappa p+1)^{-1} \beta^{-\kappa p}$. As before, we see that

$$
\Upsilon(x, \xi)=|\xi|-\vartheta(x), \quad \Upsilon(x, \xi)=\sum_{j=1}^{N} \vartheta_{j}(x) \xi_{j}-\vartheta(x), \quad \xi=\left(\xi_{1}, \ldots, \xi_{N}\right)
$$

are suitable choices for $\Upsilon$. Hence, our results can be applied to the sets

$$
\begin{aligned}
& K=\left\{u \in W^{1, p}(\Omega):|\nabla u| \leq \vartheta \quad \text { a.e. in } \Omega\right\}, \\
& K=\left\{u \in W^{1, p}(\Omega): \sum_{j=1}^{N} \vartheta_{j} \frac{\partial u}{\partial x_{j}} \leq \vartheta \quad \text { a.e. in } \Omega\right\} .
\end{aligned}
$$

\section{The case $V=W^{1, p(\cdot)}(\Omega)$ Via De Giorgi iteration}

In this section we will provide a priori bounds for (1.1) by applying De Giorgi's iteration technique if $p$ is a continuous function on $\bar{\Omega}$. Our assumptions in that case read as follows:

(H2) The mappings $A: \Omega \times \mathbb{R} \times \mathbb{R}^{N} \rightarrow \mathbb{R}^{N}$ and $F: \Omega \times \mathbb{R} \times \mathbb{R}^{N} \rightarrow \mathbb{R}$ are supposed to satisfy a Carathéodory condition while $x \mapsto j_{1}(x, s), x \mapsto j_{2}(x, s)$ are measurable for all $s \in \mathbb{R}$ and $s \mapsto j_{1}(x, s), s \mapsto j_{2}(x, s)$ are locally Lipschitz for a.a. $x \in \Omega$ and for a.a. $x \in \partial \Omega$, respectively. Furthermore, it is assumed the following:

(i) $A(x, s, \xi) \cdot \xi \geq a_{1}|\xi|^{p(x)}-a_{2}|s|^{q_{1}(x)}-a_{3} \quad$ for a.a. $x \in \Omega$;

(ii) $|A(x, s, \xi)| \leq a_{4}|\xi|^{p(x)-1}+a_{5}|s|^{q_{1}(x) \frac{p(x)-1}{p(x)}}+a_{6} \quad$ for a.a. $x \in \Omega$;

(iii) $|F(x, s, \xi)| \leq b_{1}|\xi|^{p(x) \frac{q_{1}(x)-1}{q_{1}(x)}}+b_{2}|s|^{q_{1}(x)-1}+b_{3} \quad$ for a.a. $x \in \Omega$;

(iv) $|\eta| \leq c_{1}|s|^{q_{1}(x)-1}+c_{2} \quad$ for a.a. $x \in \Omega$, for all $\eta \in \partial j_{1}(x, s)$;

(v) $|\tau| \leq d_{1}|s|^{q_{2}(x)-1}+d_{2}, \quad$ for a.a. $x \in \partial \Omega$, for all $\tau \in \partial j_{2}(x, s)$; 
and for all $s \in \mathbb{R}$, and all $\xi \in \mathbb{R}^{N}$ with positive constants $a_{i}, b_{j}, c_{k}, d_{l}$ $(i \in\{1, \ldots, 6\}, j \in\{1, \ldots, 3\}, k, l \in\{1,2\})$ and fixed functions $p, q_{1} \in C(\bar{\Omega})$, $q_{2} \in C(\partial \Omega)$ such that $1<\inf _{x \in \bar{\Omega}} p(x)$ and

$$
p(x) \leq q_{1}(x)<p^{*}(x), x \in \bar{\Omega}, \quad p(x) \leq q_{2}(x)<p_{*}(x), x \in \partial \Omega,
$$

with the critical exponents

$$
p^{*}(x)=\left\{\begin{array}{ll}
\frac{N p(x)}{N-p(x)} & \text { if } p(x)<N, \\
+\infty & \text { if } p(x) \geq N,
\end{array} \quad p_{*}(x)= \begin{cases}\frac{(N-1) p(x)}{N-p(x)} & \text { if } p(x)<N, \\
+\infty & \text { if } p(x) \geq N .\end{cases}\right.
$$

As in the case of constant exponents we obtain a certain growth rate of the generalized directional derivatives of the form

$$
\begin{array}{ll}
\left|j_{1}^{\circ}(x, s ; r)\right| \leq\left(c_{1}|s|^{q_{1}(x)-1}+c_{2}\right)|r| & \text { for a.a. } x \in \Omega \text { and for all } s, r \in \mathbb{R}, \\
\left|j_{2}^{\circ}(x, s ; r)\right| \leq\left(d_{1}|s|^{q_{2}(x)-1}+d_{2}\right)|r| & \text { for a.a. } x \in \partial \Omega \text { and for all } s, r \in \mathbb{R} .
\end{array}
$$

Then, the finiteness of the left-hand side in (1.1) is a consequence of the compact embedding $W^{1, p(\cdot)}(\Omega) \rightarrow L^{q_{1}(\cdot)}(\Omega)$ and the fact that the trace operator is a bounded operator from $W^{1, p(\cdot)}(\Omega)$ into $L^{q_{2}(\cdot)}(\partial \Omega)$ (see Fan-Shen-Zhao [14, Theorem 1.3] and Fan [13, Corollary 2.4]). Notice that we do not need log-Hölder continuity conditions, the variable exponents $p, q_{1}$, and $q_{2}$ are only supposed to be continuous.

We assume the following hypotheses on the set $K$.

(K3) For $u \in K$ and $\kappa>0$ there exists $t>0$ such that

$$
\varphi=u-t(u-\kappa)_{+} \in K .
$$

(K4) For $u \in K$ and $\kappa>0$ there exists $h>0$ such that

$$
\psi=u-h(u+\kappa)_{-} \in K .
$$

Since $W^{1, p(\cdot)}(\Omega)$ has lattice structure we notice that both function $\varphi$ and $\psi$ belong to $W^{1, p(\cdot)}(\Omega)$.

We start with the result on truncated energy estimates.

Proposition 4.1. Assume hypothesis (H2) and let $u \in K$ be a solution of (1.1).

(1) If condition (K3) is satisfied, then

$$
\int_{A_{\kappa}}|\nabla u|^{p(x)} d x \leq \hat{M}_{1} \int_{A_{\kappa}} u^{q_{1}(x)} d x+\hat{M}_{2} \int_{\partial A_{\kappa}} u^{q_{2}(x)} d \sigma,
$$

where

$$
A_{\kappa}=\{x \in \Omega: u(x)>\kappa\}, \quad \partial A_{\kappa}=\{x \in \partial \Omega: u(x)>\kappa\}, \quad \kappa \geq 1,
$$

and with positive constants $\hat{M}_{1}=\hat{M}_{1}\left(q_{1}, a_{1}, a_{2}, a_{3}, b_{1}, b_{2}, b_{3}, c_{1}, c_{2}\right)$ and $\hat{M}_{2}=\hat{M}_{2}\left(a_{1}, d_{1}, d_{2}\right)$.

(2) If condition (K4) is satisfied, then

$$
\int_{\tilde{A}_{\kappa}}|\nabla u|^{p(x)} d x \leq \hat{M}_{1} \int_{\tilde{A}_{\kappa}}(-u)^{q_{1}(x)} d x+\hat{M}_{2} \int_{\partial \tilde{A}_{\kappa}}(-u)^{q_{2}(x)} d \sigma,
$$

where

$\tilde{A}_{\kappa}=\{x \in \Omega:-u(x)>\kappa\}, \quad \partial \tilde{A}_{\kappa}=\{x \in \partial \Omega:-u(x)>\kappa\}, \quad \kappa \geq 1$, with the same constants $\hat{M}_{1}$ and $\hat{M}_{2}$ as in part (1). 
ON THE BOUNDEDNESS OF SOLUTIONS TO ELLIPTIC VARIATIONAL INEQUALITIES 15

Proof. Let $\kappa \geq 1$ be fixed and let $u \in K$ be a solution of (1.1). Due to (K3) we may choose $v=\varphi=u-t(u-\kappa)_{+} \in K$ in (1.1) to get

$$
\begin{aligned}
& \int_{A_{\kappa}} A(x, u, \nabla u) \cdot \nabla(t(u-\kappa)) d x \\
& \leq \int_{A_{\kappa}} F(x, u, \nabla u)(-t(u-\kappa)) d x+\int_{A_{\kappa}} j_{1}^{\circ}(x, u ;-t(u-\kappa)) d x \\
& \quad+\int_{\partial A_{\kappa}} j_{2}^{\circ}(x, u ;-t(u-\kappa)) d \sigma .
\end{aligned}
$$

Since $r \mapsto j_{k}^{\circ}(x, s ; r)$ is positively homogeneous (see [9, Proposition 2.1.1]) we may divide inequality (4.4) by $t>0$. By virtue of condition (H2)(i) we obtain for the left-hand side of (4.4)

$$
\begin{aligned}
& \int_{A_{\kappa}} A(x, u, \nabla u) \cdot \nabla(u-\kappa) d x \\
& =\int_{A_{\kappa}} A(x, u, \nabla u) \cdot \nabla u d x \\
& \geq \int_{A_{\kappa}}\left(a_{1}|\nabla u|^{p(x)}-a_{2}|u|^{q_{1}(x)}-a_{3}\right) d x \\
& \geq a_{1} \int_{A_{\kappa}}|\nabla u|^{p(x)} d x-\left(a_{2}+a_{3}\right) \int_{A_{\kappa}}|u|^{q_{1}(x)} d x,
\end{aligned}
$$

where $u^{q_{1}(x)}>u>1$ in $A_{\kappa}$ was taken into account. The first term on the righthand side can be estimated via Young's inequality with $\varepsilon \in(0,1]$ and condition (H2)(iii). This leads to

$$
\begin{aligned}
& \int_{A_{\kappa}} F(x, u, \nabla u)(-(u-\kappa)) d x \\
& \leq \int_{A_{\kappa}}\left[b_{1}|\nabla u|^{p(x) \frac{q_{1}(x)-1}{q_{1}(x)}}+b_{2}|u|^{q_{1}(x)-1}+b_{3}\right](u-\kappa) d x \\
& \leq b_{1} \int_{A_{\kappa}}\left[\varepsilon^{\frac{q_{1}(x)-1}{q_{1}(x)}}|\nabla u|^{p(x) \frac{q_{1}(x)-1}{q_{1}(x)}} \varepsilon^{-\frac{q_{1}(x)-1}{q_{1}(x)}} u\right] d x+\left(b_{2}+b_{3}\right) \int_{A_{\kappa}} u^{q_{1}(x)} d x \\
& \leq b_{1} \int_{A_{\kappa}} \varepsilon|\nabla u|^{p(x)} d x+b_{1} \int_{A_{\kappa}} \varepsilon^{-\left(q_{1}(x)-1\right)} u^{q_{1}(x)} d x \\
& \quad+\left(b_{2}+b_{3}\right) \int_{A_{\kappa}} u^{q_{1}(x)} d x \\
& \leq \varepsilon b_{1} \int_{A_{\kappa}}|\nabla u|^{p(x)} d x+\left(b_{1} \varepsilon^{-\left(q_{1}^{+}-1\right)}+b_{2}+b_{3}\right) \int_{A_{\kappa}} u^{q_{1}(x)} d x,
\end{aligned}
$$

where $q_{1}^{+}=\max _{\bar{\Omega}} q_{1}$. By means of hypothesis (H2)(iv) (see also (4.2)) the second term on the right-hand side of (4.4) gives

$$
\begin{aligned}
\int_{A_{\kappa}} j_{1}^{\circ}(x, u ;-(u-\kappa)) d x & \leq \int_{A_{\kappa}}\left(c_{1}|u|^{q_{1}(x)-1}+c_{2}\right)(u-\kappa) d x \\
& \leq\left(c_{1}+c_{2}\right) \int_{A_{\kappa}} u^{q_{1}(x)} d x .
\end{aligned}
$$


Finally, due to (H2)(v) (see also (4.3)), we arrive

$$
\begin{aligned}
\int_{\partial A_{\kappa}} j_{2}^{\circ}(x, u ;-(u-\kappa)) d \sigma & \leq \int_{\partial A_{\kappa}}\left(d_{1}|u|^{q_{2}(x)}+d_{2}\right)(u-\kappa) d \sigma \\
& \leq\left(d_{1}+d_{2}\right) \int_{\partial A_{\kappa}} u^{q_{2}(x)} d \sigma .
\end{aligned}
$$

Combining (4.4)-(4.8) and choosing $\varepsilon=\min \left(1, \frac{a_{1}}{2 b_{1}}\right)$ yields

$$
\begin{aligned}
& \frac{a_{1}}{2} \int_{A_{\kappa}}|\nabla u|^{p(x)} d x \\
& \leq\left(a_{2}+a_{3}+b_{1} \varepsilon^{-\left(q_{1}^{+}-1\right)}+b_{2}+b_{3}+c_{1}+c_{2}\right) \int_{A_{\kappa}} u^{q_{1}(x)} d x \\
& \quad+\left(d_{1}+d_{2}\right) \int_{\partial A_{\kappa}} u^{q_{2}(x)} d \sigma .
\end{aligned}
$$

Now we may divide (4.9) by $\frac{a_{1}}{2}>0$ which yields claim (1).

In order to prove part (2) we take $v=\psi=u-h(u+\kappa)_{-} \in K$ as test function in (1.1). This leads to

$$
\begin{aligned}
& \quad h \int_{\tilde{A}_{\kappa}} A(x, u, \nabla u) \cdot \nabla(u+\kappa) d x \\
& \leq-h \int_{\tilde{A}_{\kappa}} F(x, u, \nabla u)(u+\kappa) d x+h \int_{\tilde{A}_{\kappa}} j_{1}^{\circ}(x, u ;-(u+\kappa)) d x \\
& \quad+h \int_{\partial \tilde{A}_{\kappa}} j_{2}^{\circ}(x, u ;-(u+\kappa)) d \sigma .
\end{aligned}
$$

Dividing again by $h>0$ and applying the structure conditions in (H2)(i), (iii)-(v) we obtain the estimate in (2).

Now we can state our main result on upper and lower bounds for solutions of (1.1).

Theorem 4.2. Assume hypothesis (H2) and let $u \in K$ be a solution of (1.1). Then there exists a constant $C_{2}>0$ such that the following assertions hold.

(1) If condition (K3) is satisfied, then

$$
\underset{x \in \Omega}{\operatorname{ess} \sup } u(x) \leq C_{2} \text {. }
$$

(2) If condition (K4) is satisfied, then

$$
\underset{x \in \Omega}{\operatorname{ess} \inf } u(x) \geq-C_{2} \text {. }
$$

Proof. The proof can exactly be done as in [32, Theorem 1.1] where Lemma 3.1 and 3.2 in [32] have to be replaced by Proposition 4.1.

Analogous to Section 3 we have the corresponding result on the boundedness of solutions of (1.1).

Corollary 4.3. Let the conditions in (H2), (K3), and (K4) be satisfied and let $u \in K$ be a solution of (1.1). Then there holds

$$
\underset{x \in \Omega}{\operatorname{ess} \sup }|u(x)| \leq C_{2} \text {, }
$$

with the same constant $C_{2}$ stated in Theorem 4.2. 
Remark 4.4. As proved in [32] the constant $C_{2}$ given in Theorem 4.2 and Corollary 4.3 can be characterized through

$$
C_{2}=2 \max \left(1, \hat{M}_{3}\left[\int_{\Omega} u_{+}^{q_{1}(x)} d x+\int_{\partial \Omega} u_{+}^{q_{2}(x)} d \sigma\right]^{\hat{M}_{4}}\right)
$$

with positive constants $\hat{M}_{3}=\hat{M}_{3}\left(p, q_{1}, q_{2}, a_{1}, a_{2}, a_{3}, b_{1}, b_{2}, b_{3}, c_{1}, c_{2}, d_{1}, d_{2}, N, \Omega\right)$ and $\hat{M}_{4}=\hat{M}_{4}\left(p, q_{1}, q_{2}\right)$. As already mentioned in the constant exponent case the growth condition for $A: \Omega \times \mathbb{R} \times \mathbb{R}^{N} \rightarrow \mathbb{R}^{N}$ (see (H2)(ii)) is not needed in the proof of Theorem 4.2.

Remark 4.5. The proof of Theorem 1.1 in [32] is mainly based on the localization method combined with an appropriate choice of the partition of unity. Since $\bar{\Omega}$ is compact we find, for any $R>0$, a finite open cover $\left\{B_{i}(R)\right\}_{i=1, \ldots, m}$ of balls $B_{i}:=B_{i}(R)$ with radius $R$ such that $\bar{\Omega} \subset \bigcup_{i=1}^{m} B_{i}(R)$. Because of the continuity of $p, q_{1}, q_{2}$ and due to (4.1) we may take $R>0$ small enough such that

$$
p_{i}^{+} \leq q_{1, i}^{+}<\left(p_{i}^{-}\right)^{*}, \quad p_{i}^{+} \leq q_{2, i}^{+}<\left(p_{i}^{-}\right)_{*}, \quad i=1, \ldots, m,
$$

where

$$
\begin{array}{ll}
p_{i}^{+} & =\max _{x \in B_{i} \cap \bar{\Omega}} p(x), \quad q_{1, i}^{+}=\max _{x \in B_{i} \cap \bar{\Omega}} q_{1}(x), \\
p_{i}^{-} & =\min _{x \in B_{i} \cap \bar{\Omega}} p(x), \quad q_{2, i}^{+}=\max _{x \in B_{i} \cap \partial \Omega} q_{2}(x),
\end{array}
$$

and $\left(p_{i}^{-}\right)^{*},\left(p_{i}^{-}\right)_{*}$ denote the usual critical exponents of $p_{i}^{-}$. Then, we can choose a partition of unity $\left\{\xi_{i}\right\}_{i=1}^{m} \subset C_{0}^{\infty}\left(\mathbb{R}^{N}\right)$ with respect to the open cover $\left\{B_{i}(R)\right\}_{i=1, \ldots, m}$. That means, we have

$$
\operatorname{supp} \xi_{i} \subset B_{i}, \quad 0 \leq \xi_{i} \leq 1, \quad i=1, \ldots, m, \quad \text { and } \quad \sum_{i=1}^{m} \xi_{i}=1 \text { on } \bar{\Omega} .
$$

In fact, the idea is to treat problems involving nonstandard growth conditions as problems with constant exponent growth rates. It arises the open question what happens if $p$ is not continuous, but essentially bounded. In this case the ideas above cannot be applied.

Remark 4.6. In contrast to De Giorgi's iteration it seems that Moser's iteration is less suitable for problems with variable exponents concerning global a priori bounds. With view to the assumptions in (K1) and (K2), we see that $p$ occurs as exponent in the test function. This makes the corresponding gradient more complicated and it is not clear for the author if this method works in the variable exponent case even if $K=W^{1, p(\cdot)}(\Omega)$ and $j_{k}(k=1,2)$ smooth.

Now we give sufficient conditions for the set $K$ satisfying the conditions in (K3) and (K4).

\section{Lemma 4.7.}

(1) If $K$ contains the positive constant functions and if $K$ satisfies the condition $K \wedge K \subset K$, then the hypothesis (K3) is satisfied with $t=1$.

(2) If $K$ contains the negative constant functions and if $K$ satisfies the condition $K \vee K \subset K$, then the hypothesis (K4) is satisfied with $h=1$.

(3) If $K$ contains the constant functions and if $K$ has lattice structure, then the hypotheses (K3) and (K4) are satisfied with $t=h=1$. 
Proof. Let $u \in K$. Since $K$ is closed under " $\wedge$ " we obtain, for $\kappa \in K$ with $\kappa>0$,

$$
\min (u, \kappa)=u-(u-\kappa)_{+} \in K \text {. }
$$

Hence, condition (K3) is satisfied for $t=1$. This proves (1). Similarly, we have, for $u \in K$ and $-\kappa \in K$ with $\kappa>0$,

$$
\max (u,-\kappa)=u-(u+\kappa)_{-} \in K,
$$

as $K$ is closed under " $\vee$ ". Then, condition (K4) is fulfilled for $h=1$ which yields the assertion in (2). The last part follows directly from (1) and (2).

Let us consider some examples.

Example 4.8. Let $\vartheta: \Omega \rightarrow \mathbb{R}$ be a given function with $\vartheta \geq 0$ a.e. in $\Omega$ and consider the sets

$$
\begin{aligned}
& K=\left\{v \in W^{1, p(\cdot)}(\Omega): v \leq \vartheta \quad \text { a.e. in } \Omega\right\}, \\
& K=\left\{v \in W^{1, p(\cdot)}(\Omega): v \geq-\vartheta \quad \text { a.e. in } \Omega\right\}, \\
& K=\left\{v \in W^{1, p(\cdot)}(\Omega):|\nabla v| \leq \vartheta \quad \text { a.e. in } \Omega\right\} .
\end{aligned}
$$

We observe that these sets have lattice structure, that means, they fulfill (2.5). Moreover, the first set contains the negative constant functions, the second set the positive constant functions and the last one the constant functions. Hence, the assumptions of Lemma 4.7 are satisfied and Theorem 4.2 can be applied to these sets.

As a special case of the second set we have the cone of nonnegative elements of $W^{1, p(\cdot)}(\Omega)$, that is

$$
K_{\geq 0}=\left\{v \in W^{1, p(\cdot)}(\Omega): v \geq 0 \quad \text { a.e. in } \Omega\right\} .
$$

It is clear that $K_{\geq 0}$ has lattice structure and contains the positive constant functions. Hence, Lemma 4.7(1) implies that $K_{\geq 0}$ satisfies (K3) and from Theorem 4.2(1) we infer that every solution $u \in K_{\geq 0}$ of $(1.1)$ is bounded from above. Since $K_{\geq 0}$ is bounded from below by zero, we have that $u \in L^{\infty}(\Omega)$.

\section{REFERENCES}

[1] R. A. Adams, "Sobolev spaces", Academic Press, New York-London, 1975.

[2] G. Bonanno, D. Motreanu, P. Winkert, Variational-hemivariational inequalities with small perturbations of nonhomogeneous Neumann boundary conditions, J. Math. Anal. Appl. 381 (2011), no. 2, 627-637.

[3] G. Bonanno, P. Winkert, Multiplicity results to a class of variational-hemivariational inequalities, Topol. Methods Nonlinear Anal., to appear.

[4] S. Carl, Elliptic variational inequalities with discontinuous multi-valued lower order terms, Adv. Nonlinear Stud. 13 (2013), no. 1, 55-78.

[5] S. Carl, Equivalence of some multi-valued elliptic variational inequalities and variationalhemivariational inequalities, Adv. Nonlinear Stud. 11 (2011), no. 2, 247-263.

[6] S. Carl, Parameter-dependent variational-hemivariational inequalities and an unstable degenerate elliptic free boundary problem, Nonlinear Anal. Real World Appl. 12 (2011), no. 6, 3185-3198.

[7] S. Carl, V. K. Le, D. Motreanu, "Nonsmooth variational problems and their inequalities", Springer, New York, 2007.

[8] K. C. Chang, Variational methods for nondifferentiable functionals and their applications to partial differential equations, J. Math. Anal. Appl. 80 (1981), no. 1, 102-129.

[9] F. H. Clarke, "Optimization and Nonsmooth Analysis", Society for Industrial and Applied Mathematics (SIAM), Philadelphia, 1990.

[10] E. DiBenedetto, "Degenerate Parabolic Equations", Springer, New York, 1993. 
[11] L. Diening, P. Harjulehto, P. Hästö, M. Růžička, "Lebesgue and Sobolev spaces with variable exponents", Springer, Heidelberg, 2011.

[12] P. Drábek, A. Kufner, F. Nicolosi, "Quasilinear elliptic equations with degenerations and singularities", Walter de Gruyter \& Co., Berlin, 1997.

[13] X. Fan, Boundary trace embedding theorems for variable exponent Sobolev spaces, J. Math. Anal. Appl. 339 (2008), no. 2, 1395-1412.

[14] X. Fan, J. Shen, D. Zhao, Sobolev embedding theorems for spaces $W^{k, p(x)}(\Omega)$, J. Math. Anal. Appl. 262 (2001), no. 2, 749-760.

[15] X. Fan, D. Zhao, On the spaces $L^{p(x)}(\Omega)$ and $W^{m, p(x)}(\Omega)$, J. Math. Anal. Appl. 263 (2001), no. $2,424-446$.

[16] Y. S. Gorban, A. A. Kovalevsky, On the Boundedness of Solutions of Degenerate Anisotropic Elliptic Variational Inequalities, Results Math. 65 (2014), no. 1-2, 121-142.

[17] J. Heinonen, T. Kilpeläinen, O. Martio, "Nonlinear potential theory of degenerate elliptic equations", Dover Publications Inc., Mineola, NY, 2006.

[18] S. Hu, N. S. Papageorgiou, Nonlinear Neumann equations driven by a nonhomogeneous differential operator, Commun. Pure Appl. Anal. 10 (2011), no. 4, 1055-1078.

[19] J. Ježková, Boundedness and pointwise differentiability of weak solutions to quasi-linear elliptic differential equations and variational inequalities, Comment. Math. Univ. Carolin. 35 (1993), no. 1, 63-80.

[20] A. Kovalevsky, F. Nicolosi, Boundedness of solutions of degenerate nonlinear elliptic variational inequalities, Nonlinear Anal. 35 (1999), no. 8, 987-999.

[21] A. Kovalevsky, F. Nicolosi, Boundedness of solutions of variational inequalities with nonlinear degenerated elliptic operators of high order, Appl. Anal. 65 (1997), no. 3-4, 225-249.

[22] O. Kováčik, J. Rákosník, On spaces $L^{p(x)}$ and $W^{k, p(x)}$, Czechoslovak Math. J. 41(116) (1991), no. 4, 592-618.

[23] S. Kyritsi, N. S. Papageorgiou, Solutions for hemivariational and variational-hemivariational inequalities, Dyn. Contin. Discrete Impuls. Syst. Ser. B Appl. Algorithms 18 (2011), no. 4, $521-541$.

[24] O. A. Ladyženskaja, V. A. Solonnikov, N. N. Ural'ceva, Linear and Quasilinear Equations of Parabolic Type, American Mathematical Society, Providence, R.I., 1968.

[25] V. K. Le, On a sub-supersolution method for variational inequalities with Leray-Lions operators in variable exponent spaces, Nonlinear Anal. 71 (2009), no. 7-8, 3305-3321.

[26] D. Motreanu, V. Rădulescu, "Variational and non-variational methods in nonlinear analysis and boundary value problems", Kluwer Academic Publishers, Dordrecht, 2003.

[27] D. Motreanu, P. Winkert, Variational-hemivariational inequalities with nonhomogeneous Neumann boundary condition, Matematiche (Catania) 65 (2010), no. 2, 109-119.

[28] H. Triebel, "Theory of function spaces", Akademische Verlagsgesellschaft Geest \& Portig K.-G., Leipzig, 1983.

[29] H. Triebel, "Theory of function spaces II", Birkhäuser Verlag, Basel, 1992.

[30] P. Winkert, Constant-sign and sign-changing solutions for nonlinear elliptic equations with Neumann boundary values, Adv. Differential Equations 15 (2010), no. 5-6, 561-599.

[31] P. Winkert, $L^{\infty}$-estimates for nonlinear elliptic Neumann boundary value problems, NoDEA Nonlinear Differential Equations Appl. 17 (2010), no. 3, 289-302.

[32] P. Winkert, R. Zacher, A priori bounds for weak solutions to elliptic equations with nonstandard growth, Discrete Contin. Dyn. Syst. Ser. S 5 (2012), no. 4, 865-878.

(P. Winkert) Technische Universität Berlin, Institut für Mathematik, Strasse des 17. Juni 136, 10623 Berlin, Germany

E-mail address: winkert@math.tu-berlin.de 